\title{
Mechanism of monosynaptic reflex reinforcement during Jendrassik manoeuvre in man
}

\author{
B. B USS E L, C. M O R I N, A N D E. P I E R R O T - D E S E I L L I G N Y \\ From the Service de Rééducation, Hôpital de la Salpêtrière, Paris, France
}

SUMMARY The facilitating effect of the Jendrassik manoeuvre on monosynaptic reflexes in man has been studied by comparing $\mathrm{H}$ and $\mathrm{T}$ reflexes of the soleus and by blocking the effects of spindle activation by ischaemia of the leg. The Jendrassik manoeuvre equally enhances $H$ and $T$ reflexes provided that the test reflexes are small. The $H$ reflex remains facilitated when the spindle activation cannot affect the soleus $\alpha$-motoneurones-that is, when the Ia afferent nerve fibres from the soleus are blocked by ischaemia. It is concluded that the facilitating effect of the Jendrassik manoeuvre on the $\alpha$-motoneurones is not predominantly routed via the $\gamma$-loop.

The facilitating effect of the Jendrassik manoeuvre on tendon reflexes in man is well-known but the mechanism for this reinforcement is not yet clear. It is not known whether this enhancement is predominantly due to a direct facilitation of $\alpha$-motoneurones or to an increase in spindle activity induced by $\gamma$-activation. Controversial results have been obtained by experimenters using either reflex or microneuronographic methods. Reflex studies compared the effects of the Jendrassik manoeuvre on the Achilles tendon reflex and on the $\mathrm{H}$ reflex of the soleus. This electrically evoked $\mathrm{H}$ reflex is also a monosynaptic reflex of the soleus, but it bypasses the soleus spindles, and, therefore, does not depend directly on $\gamma$-fibre activity. Sommer (1940), Paillard (1955), and Buller and Dornhorst (1957) claimed that the $\mathrm{H}$ reflex was less facilitated than the Achilles tendon (T) reflex. Different results have been obtained by Landau and Clare (1964) who found that the Jendrassik manoeuvre affected both $\mathrm{H}$ and $\mathrm{T}$ reflexes equally. The observed discrepancies are certainly due to technical differences regarding the strength of the reinforcing manoeuvre, the temporal relationship between the beginning of the handgrip and the test reflex, and the amplitude of the $\mathrm{H}$ and $\mathrm{T}$ test reflexes before the Jendrassik manoeuvre. Clare and Landau (1964) argued strongly against the gamma hypothesis when they found that the $\mathrm{H}$ reflex enhancement

Address for correspondence and reprint requests: Dr B. Bussel, Service de Rééducation, Hôpital de la Salpêtrière, 47, Bd de l'Hôpital, 75634 Paris Cedex 13, France.

Accepted 15 July 1977 persisted after fusimotor block with procaine. However, the use of microneuronographic techniques, which permit monitoring of the $\gamma$ activity by recording from the Ia afferent fibres from the spindles has raised the problem once more. Burgo et al. (1974) found an increase of spindle activity in relaxed muscles during contraction of remoteo muscles and thought, therefore, that $\gamma$-fibre activity was responsible for the enhancement of tendon reflexes during the Jendrassik manoeuvre. On the contrary, Hagbarth et al. (1975) have shown that the spindle activation is always accompanied by a very slight contraction of skeletomotor fibres which implies $\alpha$-activation. Therefore, for them, $\gamma$-activation during the Jendrassik manoeuvre is never isolated.

In this study we have observed the effect of the Jendrassik manoeuvre in man upon the $T$ and $H$ reflexes of the soleus. We have studied the effects observed at the beginning of the handgrip and during a sustained handgrip; the latter situation was first described by Jendrassik (1883). In addition, we have compared the effect of the Jendrassik manoeuvre upon the soleus $\mathrm{H}$ reflex before and during an ischaemic block of large diameter afferent nerve fibres from the leg; the disappearance of the Achilles tendon reflex during ischaemia proved the block of Ia afferent fibres from the soleus.

\section{Methods}

This investigation was performed in five healthy adult volunteers (26-41 years old). The subjects were comfortably seated in an armchair with hip 
and knee flexed at $120^{\circ}$. We studied the variations of the soleus $\mathrm{H}$ reflex and Achilles tendon reflex during the Jendrassik manoeuvre before and during ischaemia of the leg. The reflex responses were measured in terms of muscle action potentials recorded by two surface electrodes placed $15 \mathrm{~mm}$ apart on the soleus muscle below the gastrocnemius. The EMG responses were recorded on a magnetic tape and analysed by a Nova 1200 computer. The soleus $\mathrm{H}$ reflex was obtained by stimulation of the posterior tibial nerve at the popliteal fossa with single rectangular electrical pulses of $1 \mathrm{~ms}$ duration delivered through a monopolar electrode. The tendon reflex was elicited with an electromagnetic hammer (Bruel and Kjaer 4809), which produced a stretch of the soleus of $5 \mathrm{~ms}$ duration and $8 \mathrm{~mm}$ amplitude. The strength of the tap delivered by the hammer was adjusted so that the amplitude of the control $T$ reflex was half its maximum value. The intensity of the $\mathrm{H}$ reflex stimulation was fixed so that the resulting $H$ reflex had approximately the same amplitude as the control $\mathrm{T}$ reflex. The Jendrassik manoeuvre was performed in two ways.

(1) The subjects were instructed to perform a brisk and strong handgrip of both hands (duration of one second, in response to a visual stimulus. This stimulus was delivered every three seconds. The test reflex was evoked $400 \mathrm{~ms}$ after the onset of the handgrip. This test reflex was alternatively an $\mathrm{H}$ reflex or a tendon reflex. Each series of 20 conditioned reflexes was followed by 20 additional reflexes evoked at rest. These control reflexes were preceded by the same visual stimulus.

(2) The subjects were asked to perform a sustained handgrip of both hands and to control the force of their handgrip, which was possible by using a dynamometer. Three levels of force (maximal, half maximal, quarter maximal) were used. Each series of 20 (or 10) unconditioned reflexes was followed by three groups of conditioned reflexes, each group being obtained with a given strength of handgrip. In both situations, the mean amplitude of the $\mathrm{H}$ and $\mathrm{T}$ reflexes was calculated for each period of $30 \mathrm{~s}$, a time during which five $T$ reflexes and five $H$ reflexes were evoked. This mean amplitude was expressed as a percentage of the maximum $\mathbf{M}$ direct response of the soleus.

Ischaemia of the leg was obtained by placing a sphygmomanometer cuff at the high part of the

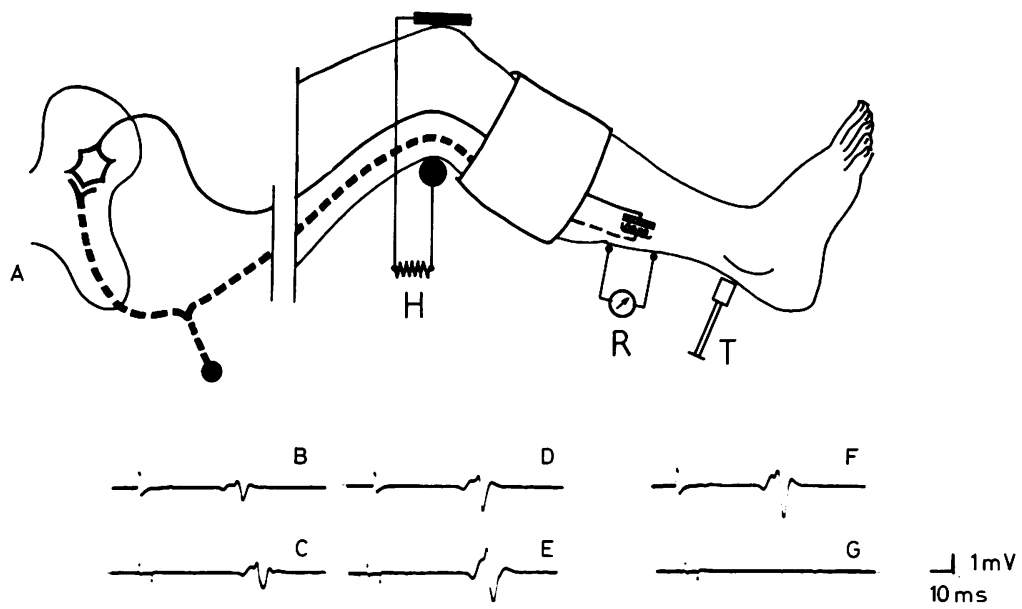

Fig. 1 Experimental design for producing ischaemia of the lower part of the leg. $R$ symbolises the recording systems, $H$ the $H$ reflex stimulation, and $T$ the hammer used to produce tendon taps. The pathway of the soleus monosynaptic reflex is also shown. Motor nerve fibres are represented by continuous lines, afferent fibres by thick interrupted lines for normally conducting segments and thin interrupted lines for the segments blocked by ischaemia. $B-G$ : $E M G$ recordings of $H$ (upper line) and $T$ (lower line) reflexes. $B-C$ : control responses. $D-E$ : responses obtained during reinforcement manoeuvre. $F-G$ : responses obtained during reinforcement manoeuvre, group I afferent fibres being blocked by ischaemia. 
leg, but below the site of the $H$ reflex stimulation (Fig. 1A). The sphygmomanometer cuff was inflated at $300 \mathrm{~mm} \mathrm{Hg}$.

\section{Results}

EFFECTS OF TWO KINDS OF REINFORCING

MANOEUVRE ON H AND T REFLEXES

Figure 2 shows the results obtained in one subject during a brisk and strong handgrip. The same observations were made in all subjects. The amplitude of $H$ and $T$ reflexes was identical before ischaemia and during the early minutes of ischaemia. Therefore, the first 15 minutes of the ischaemia could be considered as a control period. During this control period, it was possible to keep the amplitude of both $\mathrm{H}$ and $\mathrm{T}$ reflexes equal to approximately $20 \%$ of the maximum $\mathrm{M}$ response
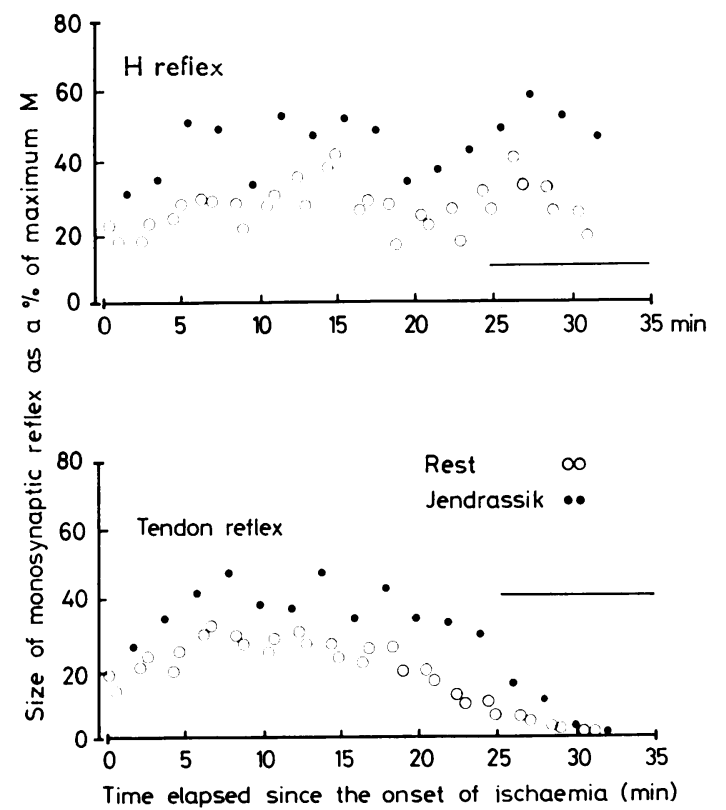

Fig. 2 Effect of ischaemia of the leg on soleus monosynaptic reflex facilitation obtained by a strong and brisk handgrip. Each symbol represents the mean value of five $\mathrm{H}$ reflexes (upper graph) or five Achilles tendon reflexes (lower graph). These reflexes were obtained at rest $(O)$ and during a brisk and strong handgrip (๑). Abscissa: time elapsed since the onset of ischaemia; ordinate: size of the monosynaptic reflex expressed as a percentage of maximum direct motor response. The horizontal bar indicates the time interval during which the tendon reflex is reduced and then abolished. of the soleus. Figure 2 shows that there was an increase of about $50 \%$ in the amplitude of both $\mathrm{H}$ and $\mathrm{T}$ reflexes with the Jendrassik manoeuvre, and that the facilitation of the Achilles reflex was no stronger than that of the $\mathrm{H}$ reflex. Similar results were obtained in all subjects. The amplitude of the facilitated reflexes varied among the subjects between $122 \%$ and $185 \%$ of their control value for the $\mathrm{H}$ reflex (Fig. 1B and D), and between $140 \%$ and $196 \%$ for the tendon reflex (Fig. $1 \mathrm{C}$ and $\mathrm{D}$ ).

As claimed by Paillard (1955), the effect of a sustained handgrip upon $\mathrm{H}$ and $\mathrm{T}$ reflexes is much less marked than that of a brisk handgrip. For this reason, with subjects exhibiting a relatively high variability of monosynaptic reflexes, one would have to increase the number of reflexes in order to obtain significant results. However, as the period of ischaemia which can be explored (see below) is necessarily short, we have been able to study the effects of a sustained handgrip only on subjects in whom variability of the $\mathrm{H}$ and $\mathrm{T}$ reflexes was low. The results obtained in one subject are shown in Fig. 3. Throughout the control periode the $\mathrm{H}$ and $\mathrm{T}$ reflexes were usually more facilitated when obtained during a stronger handgrip. The mean value of the facilitation obtained when the handgrip was $25 \%, 50 \%$, and $100 \%$ of the maximal force was $131 \%, 152 \%$, and $150 \%$ respec tively of the corresponding $\mathrm{H}$ reflex control or $135 \%, 140 \%$, and $180 \%$ of the corresponding 1 reflex control.

EFFECTS OF ISCHAEMIA OF THE LEG ON THE H REFLEX FACILITATION OBSERVED DURING REINFORCEMENT MANOEUVRES

Twenty to 27 minutes after the onset of ischaemia, the amplitude of the Achilles tendon reflex began to diminish; it then decreased more and more. About $30 \mathrm{~min}$ after the inflation of the cuff, the test $H$ reflex and the $M$ response were no smaller than at the onset of ischaemia, the Achilles tendon reflex being completely abolished (Fig. 1G). The disappearance of the tendon reflex while the $\mathrm{H}$ and $M$ responses persist is due to the blockade of group I afferent nerve fibres below the site of the $\mathrm{H}$ reflex stimulation. This is in agreement with the results obtained by Magladery et al. (1950) who found that ischaemia of the leg blocks the group I afferent fibres before blocking the motor axons. Under these conditions, both kinds of reinforcement manoeuvre still result in a facilitated $\mathrm{H}$ reflex which varied among the subjects between $135 \%$ and $197 \%$ (Fig. 1F; right part of Figs. 2, 3). Whereas the tendon reflex was completely abolished (Fig. 1G and right part of Figs. 2, 3). 


\section{Discussion}

We have studied the effects of the Jendrassik manoeuvre on $\mathrm{H}$ and $\mathrm{T}$ reflexes of the soleus in man. The $\mathrm{H}$ and $\mathrm{T}$ test reflexes were of identical and relatively small amplitude. The Jendrassik manoeuvre increased both reflexes equally. This facilitation was much more marked at the onset of a brisk handgrip than during sustained contractions, as found by Paillard (1955). During a sustained handgrip the amount of facilitation depended on the strength of the handgrip, as observed by Bishop et al. (1968). As previously shown by Landau and Clare (1964), there was no difference between the increase of $\mathrm{H}$ and $\mathrm{T}$ reflexes provided that the size of both test reflexes

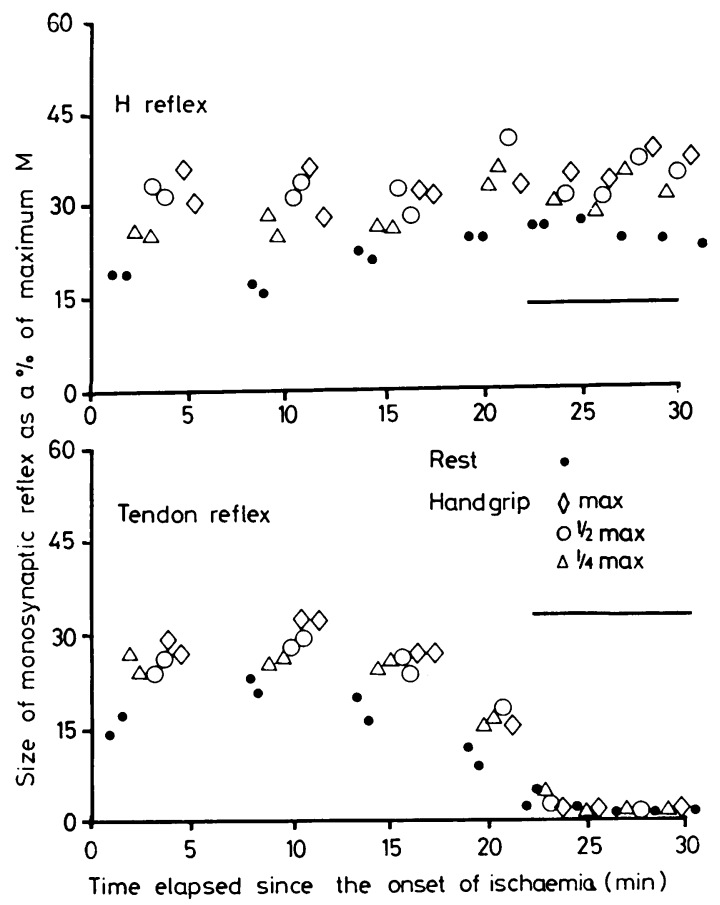

Fig. 3 Effect of ischaemia of the leg on soleus monosynaptic reflex facilitation obtained by sustained handgrip of different forces. Each symbol represents the mean value of five $H$ reflexes (upper graph) or five Achilles tendon reflexes (lower graph). These reflexes were obtained at rest (๑) and during sustained handgrips of maximal force $(\diamond)$, half maximal force $(\bigcirc)$, or quarter maximal force $(\triangle)$. Abscissa: time elapsed since the onset of ischaemia; ordinate: size of monosynaptic reflex expressed as a percentage of maximum direct motor response. The horizontal bar indicates the time interval during which the tendon reflex is reduced and then abolished. was low. This suggesis that the spindle aciivity does not play any critical role in the facilitating effect of the Jendrassik manoeuvre, and this is confirmed by the persistence of facilitation of the $\mathrm{H}$ reflex during ischaemia of the leg. This persisting facilitation is certainly not due to the painful effects of ischaemia since these effects would modify the $\mathrm{H}$ reflex in the same way whether it was reinforced or not. Our results, therefore, suggest that blocking the Ia afferent nerve fibres which discharge after $\gamma$-fibre activity does not modify the $\mathrm{H}$ reflex facilitation observed during the Jendrassik manoeuvre. Similar conclusions have been reached by Clare and Landau (1964) who blocked $\gamma$-fibres with lidocaine, a technique without objective proof of the efficiency of the block. In our experimental design, the block of the Ia fibres coming from the soleus was confirmed by the disappearance of the Achilles tendon reflex. Since the $M$ response was unchanged, it is likely that group II fibres were not blocked, since they are smaller than $\alpha$-fibres. The discharge of these fibres is also controlled by $\gamma$-activation but they are generally considered as inhibiting soleus motoneurones (Eccles and Lundberg, 1959) and can, therefore, not be responsible for the persistence of the $\mathrm{H}$ reflex facilitation.

The persistence of the facilitating effects of a Jendrassik manoeuvre, whichever way it is performed, when the fusimotor effects are eliminated by ischaemia does not exclude the possibility that $\gamma$-motoneurones are normally activated during the manoeuvre. Our results in fact demonstrate that the Jendrassik manoeuvre does not exert its effects by predominantly facilitating $\gamma$-motoneurones.

This work was supported by grants from the Pierre et Marie Curie University (Paris VI) and from the Institut National de la Santé et de la Recherche Médicale (INSERM 013, ATP 29.76.61).

\section{References}

Bishop, B., Machover, S., Johnston, R., and Anderson, M. (1968). A quantitative assessment of gamma motoneuron contribution to the Achilles tendon reflex in normal subjects. Archives of Physical Medicine and Rehabilitation, 49, 145-154.

Buller, A. J., and Dornhorst. A. C. (1957). The reinforcement of tendon reflexes. Lancet, 2, 12601262.

Burg, D., Szumski, A. J.. Struppler, A., and Velho, F. (1974). Assessment of fusimotor contribution to reflex reinforcement in humans. Journal of Neurology, Neurosurgery, and Psychiatry, 37, 10121021. 
Clare, M. H., and Landau, W. M. (1964). Fusimotor function. Part V. Reflex reinforcement under fusimotor block in normal subjects. Archives of Neurology (Chicago), 10, 123-127.

Eccles, R. M., and Lundberg, A. (1959). Synaptic action in motoneurones by afferents which may evoke the flexion reflex. Archives Italiennes de Biologie, 97, 199-221.

Hagbarth, K. E., Wallin, G., Burke, D., and Löfstedt, L. (1975). Effects of the Jendrassik manoeuvre on muscle spindle activity in man. Journal of Neurology, Neurosurgery, and Psychiatry, 38, 11431153.

Jendrassik, E. (1883). Beitrage zur Lehre von den Sehnenreflexen. Deutsches Archiv für Klinische Medizin, 33, 177-199.
Lanclau, W. M., and Clare, M. H. (1964). Fusimotor function. Part IV. Reinforcement of the H-reflex in normal subjects. Archives of Neurology (Chicago), 10, 117-122.

Magladery, J. W., McDougal, D. B., and Stoll, J. (1950). Electrophysiological studies of nerve and reflex activity in normal man. II The effects of peripheral ischaemia. Bulletin of the Johns Hopkins Hospital, 86, 291-312.

Paillard, J. (1955). Reflexes et regulations d'origine proprioceptive chez l'homme. Arnette: Paris.

Sommer, J. (1940). Periphere Bahnung von Muskeleigenreflexen als Wesen des Jendrassikschen Phänomens. Deutsche Zeitschrift für Nervenheilkunde, 150, 249-262. 